\title{
THE CHORD LENGTH DISTRIBUTION FUNCTION FOR REGULAR POLYGONS
}

\author{
H. S. HARUTYUNYAN $* * *$ AND \\ V. K. OHANYAN, ${ }^{* * * *}$ Yerevan State University
}

\begin{abstract}
In this paper we obtain an elementary expression for the chord length distribution function of a regular polygon. The formula is derived using $\delta$-formalism in Pleijel identity. In the particular cases of a regular triangle, a square, a regular pentagon, and a regular hexagon, our formula coincides with the results of Sulanke (1961), Gille (1988), Aharonyan and Ohanyan (2005), and Harutyunyan (2007), respectively.
\end{abstract}

Keywords: Chord length distribution function; Pleijel identity; regular polygons

2000 Mathematics Subject Classification: Primary 60D05; 52A22

\section{Introduction}

Let $\mathbb{G}$ be the space of all lines $g$ in the Euclidean plane $\mathbb{R}^{2}$, and let $(p, \varphi)$, the polar coordinates of the foot of the perpendicular to $g$ from the origin $O$, be the standard coordinates for a line $g \in \mathbb{G}$.

Let $\mu(\cdot)$ be the locally finite measure on $\mathbb{G}$, invariant with respect to the group of all Euclidean motions (translations and rotations). It is well known that the element of the measure up to a constant factor has the following form (see [1, p. XIII]):

$$
\mu(\mathrm{d} g)=\mathrm{d} g=\mathrm{d} p \mathrm{~d} \varphi,
$$

where $\mathrm{d} p$ is the one-dimensional Lebesgue measure and $\mathrm{d} \varphi$ is the uniform measure on the unit circle.

For each bounded convex domain $\mathbb{D}$, we denote the set of lines that intersect $\mathbb{D}$ by

$$
[\mathbb{D}]=\{g \in \mathbb{G}: g \cap \mathbb{D} \neq \varnothing\},
$$

and we have (see [1, p. 195] and [2, p. 130])

$$
\mu([\mathbb{D}])=|\partial \mathbb{D}|,
$$

where $\partial \mathbb{D}$ is the boundary of $\mathbb{D}$ and $|\partial \mathbb{D}|$ stands for the length of $\partial \mathbb{D}$.

A random line in $[\mathbb{D}]$ is one with distribution proportional to the restriction of $\mu$ to $[\mathbb{D}]$. Therefore,

$$
\mathrm{P}(A)=\frac{\mu(A)}{|\partial \mathbb{D}|} \quad \text { for any Borel set } A \subset[\mathbb{D}] .
$$

Received 1 July 2008; revision received 13 February 2009.

* Postal address: Department of Mathematics and Mechanics, Yerevan State University, 1 Alex Manoogian Street, Yerevan 0025, Armenia.

** Email address: hrach87@web.am

*** Email address: victo@aua.am 
Furthermore, let $A_{\mathbb{D}}^{y}$ be the set of lines that intersect $\mathbb{D}$, producing a chord $\chi(g)=g \cap \mathbb{D}$ of length less than $y$, i.e.

$$
A_{\mathbb{D}}^{y}=\{g \in[\mathbb{D}]:|\chi(g)| \leq y\}, \quad y \in \mathbb{R} .
$$

The distribution function for the length of a random chord $\chi$ of $\mathbb{D}$ is usually defined as

$$
F(y)=\frac{1}{|\partial \mathbb{D}|} \mu\left(A_{\mathbb{D}}^{y}\right)=\frac{1}{|\partial \mathbb{D}|} \iint_{A_{\mathbb{D}}^{y}} \mathrm{~d} \varphi \mathrm{d} p .
$$

Therefore, to obtain the chord length distribution function for a bounded convex domain $\mathbb{D}$, we have to calculate the integral on the right-hand side of (1.1). Explicit formulae for the chord length distribution functions are known only for the cases of a disc, a regular triangle [11], a rectangle [6], a regular pentagon [3], and a regular hexagon [9].

The main result of this paper is an elementary expression for the chord length distribution function of a regular polygon. The formula is derived using $\delta$-formalism in Pleijel identity. In the cases of regular triangles, squares, regular pentagons, and regular hexagons, our formula coincides with formulae available in the literature (see [3], [6], [9], and [11]).

The determination of the chord length distribution function has a long tradition of application to collections of bounded convex bodies forming structures in metals and ceramics. The series of formulae for chord length distribution functions may be of use in finding suitable models when empirical distribution functions are given (see [12, p. 116]). They also have an independent interest (see [4] and [5]). The results concerning certain infinite cylinders in which the bases of cylinders are a regular triangle or a rectangle (see [7] and [13]). Gille et al. [8] considered infinite cylinders with regular pentagonal and regular hexagonal bases.

\section{The case of a convex polygon}

Let $\mathbb{D}$ be a convex bounded polygon in the plane, and let $a_{1}, a_{2}, \ldots, a_{n}$ be the sides of $\mathbb{D}$. Then

$$
[\mathbb{D}]=\bigcup_{i<j}\left(\left[a_{i}\right] \cap\left[a_{j}\right]\right)
$$

where $\left[a_{i}\right] \cap\left[a_{j}\right]$ is the set of lines hitting both sides $a_{i}$ and $a_{j}$ of $\mathbb{D}$.

We write

$$
\begin{aligned}
F(y) & =\frac{1}{|\partial \mathbb{D}|} \sum_{i<j} \iint_{\left\{g \in\left[a_{i}\right] \cap\left[a_{j}\right]:|\chi(g)| \leq y\right\}} \mathrm{d} \varphi \mathrm{d} p \\
& =\frac{1}{|\partial \mathbb{D}|}\left(\sum_{i<j}^{I} \iint_{\left\{g \in\left[a_{i}\right] \cap\left[a_{j}\right]:|\chi(g)| \leq y\right\}} \mathrm{d} \varphi \mathrm{d} p+\sum_{i<j}^{I I} \iint_{\left\{g \in\left[a_{i}\right] \cap\left[a_{j}\right]:|\chi(g)| \leq y\right\}} \mathrm{d} \varphi \mathrm{d} p\right),
\end{aligned}
$$

where the sum ' $\sum^{I}$ ' is over all nonparallel pairs of segments $a_{i}, a_{j} \subset \partial \mathbb{D}$ and the sum ' $\sum^{I I}$, is over all parallel pairs of segments $a_{i}, a_{j} \subset \partial \mathbb{D}$.

In each of the integrals in the sum ' $\sum_{i<j}^{I}$ ', one integration can be performed by passing to $(|\chi|, \varphi)$ coordinates. We have (see [2, p. 157])

$$
\mathrm{d} g=\frac{\sin \alpha_{1} \sin \alpha_{2}}{\left|\sin \left(\alpha_{1}+\alpha_{2}\right)\right|} \mathrm{d}|\chi| \mathrm{d} \varphi,
$$

where $\alpha_{1}$ is the angle between $a_{i}$ and $\chi(g)=g \cap \mathbb{D}$, and $\alpha_{2}$ is the angle between $a_{j}$ and $\chi(g)$ $\left(g \in\left[a_{i}\right] \cap\left[a_{j}\right]\right)$, with $\alpha_{1}$ and $\alpha_{2}$ lying in one half-plane with respect to $\chi$, inside of $\mathbb{D}$. 


\section{Pleijel's identity}

Let $\mathbb{D}$ be a convex bounded polygon in the plane, and let $a_{1}, \ldots, a_{n}$ be the sides of $\mathbb{D}$. The so-called Pleijel identity for $\mathbb{D}$ is as follows (see [2, p. 156]):

$$
\int_{[\mathbb{D}]} f(|\chi(g)|) \mathrm{d} g=\int_{\mathbb{G}} f^{\prime}(|\chi|)|\chi| \cot \alpha_{1} \cot \alpha_{2} \mathrm{~d} g+\sum_{i=1}^{n} \int_{0}^{\left|a_{i}\right|} f(u) \mathrm{d} u,
$$

where $f(x)$ is a function with continuous first derivative $f^{\prime}(x), \alpha_{1}$ and $\alpha_{2}$ are the angles between $\partial \mathbb{D}$ and $g$ at the endpoints of $\chi(g)=g \cap \mathbb{D}$ which lie in one half-plane with respect to $\chi$, inside of $\mathbb{D}$, and $\left|a_{i}\right|$ is the length of $a_{i}, i=1, \ldots, n$.

It was shown in [2, p. 156] that identity (3.1) is useful in calculating the chord length distribution function for the case of bounded convex polygons. If in (3.1) we formally set

$$
f_{y}(u)= \begin{cases}0 & \text { if } u \leq y \\ 1 & \text { if } u>y\end{cases}
$$

then the left-hand side integral in (3.1) will equal

$$
\mu\{g \in[\mathbb{D}]:|\chi(g)|>y\},
$$

i.e. the invariant measure of the set of chords of $\mathbb{D}$ whose length exceeds $y$. The derivative of $f_{y}(u)$ should be replaced by Dirac's $\delta$-function concentrated at $y$ (see [2, p. 156]). Therefore, we obtain

$$
\begin{aligned}
{[1-F(y)]|\partial \mathbb{D}|=} & \sum_{i<j}^{I} \iint_{\left[a_{i}\right] \cap\left[a_{j}\right]} \delta(|\chi|-y)|\chi| \cot \alpha_{1} \cot \alpha_{2} \mathrm{~d} g \\
& +\sum_{i<j}^{I I} \iint_{\left[a_{i}\right] \cap\left[a_{j}\right]} \delta(|\chi|-y)|\chi| \cot \alpha_{1} \cot \alpha_{2} \mathrm{~d} g+\sum_{i=1}^{n}\left(\left|a_{i}\right|-y\right)^{+},
\end{aligned}
$$

where $x^{+}=x$ if $x>0$ and 0 otherwise.

For any continuous function $f$, we have (see [10])

$$
\int_{\mathbb{R}^{n}} \delta(x-y) f(x) \mathrm{d} x=f(y) .
$$

For each nonparallel pair $a_{i}$ and $a_{j}$, we make the change of variables $(p, \varphi) \rightarrow(|\chi|, \varphi)$, and using (2.1) and (3.2), we obtain

$$
\iint_{\left[a_{i}\right] \cap\left[a_{j}\right]} \delta(|\chi|-y)|\chi| \cot \alpha_{1} \cot \alpha_{2} \mathrm{~d} g=\frac{y}{\sin \gamma_{i j}} \int_{\Phi_{i j}(y)} \sin \varphi \sin \left(\gamma_{i j}-\varphi\right) \mathrm{d} \varphi,
$$

where $\gamma_{i j}$ is the angle between nonparallel sides $a_{i}$ and $a_{j}$ (or their continuations), $\varphi$ is the angle between direction $\varphi$ and the $a_{i}$ direction, $i<j$, and

$$
\Phi_{i j}(y)=\left\{\varphi \text { : a chord joining } a_{i} \text { and } a_{j} \text { exists with direction } \varphi \text { and length } y\right\} .
$$

Note that $\Phi_{i j}(y)$ is a subset in the space of directions in the plane; moreover, in the right-hand side integral, $\Phi_{i j}(y)$ corresponds to the set of angles, where the reference direction coincides 
with the direction of segment $a_{i}, i<j$, and the reference origin coincides with the intersection of the lines containing $a_{i}$ and $a_{j}$.

Moreover, for parallel sides $a_{i}$ and $a_{j}$ (i.e. $a_{i}, a_{j} \in \sum_{i<j}^{I I}$ ), we have

$$
\begin{aligned}
\sum_{i<j}^{I I} & \iint_{\left[a_{i}\right] \cap\left[a_{j}\right]} \delta(|\chi|-y)|\chi| \cot \alpha_{1} \cot \alpha_{2} \mathrm{~d} g \\
& =-\sum_{i<j}^{I I} I_{i j}(y) \int \delta(|\chi|-y)|\chi| h\left(\varphi_{\chi}\right) \tan ^{2} \varphi_{\chi} \frac{\mathrm{d} \varphi_{\chi}}{\mathrm{d}|\chi|} \mathrm{d}|\chi| \\
& =-\sum_{i<j}^{I I} I_{i j}(y) h\left(\varphi_{y}\right) \tan ^{2} \varphi_{y} \frac{b_{i j}}{\sqrt{y^{2}-b_{i j}^{2}}},
\end{aligned}
$$

where $\varphi_{y}$ is either $\arccos \left(b_{i j} / y\right)$ or $2 \pi-\arccos \left(b_{i j} / y\right), b_{i j}$ is the distance between the parallel segments $a_{i}$ and $a_{j}$ (i.e. the distance between the lines containing $a_{i}$ and $a_{j}$ ), and the indicator $I_{i j}(y)=\mathbf{1}$ (the length of the shortest chord hitting $a_{i}$ and $a_{j}$ is less than or equal to $y$ which in turn is less than or equal to the length of the longest chord hitting $a_{i}$ and $a_{j}$ ), where $\mathbf{1}(A)$ is the indicator function of the event $A$, i.e. $\mathbf{1}(A)=1$ if $A$ has occurred and 0 otherwise. Furthermore, $h(\varphi) \neq b_{i j}$ is the height of the maximal parallelogram with two sides equal to $\chi(\varphi)=g(\varphi) \cap \mathbb{D}$ and $g(\varphi) \in\left[a_{i}\right] \cap\left[a_{j}\right](g(\varphi)$ is a line with $\varphi$-direction), and the other two sides lying on the parallel sides $a_{i}$ and $a_{j}$,

$$
h\left(\varphi_{\chi}\right)=h\left(\arccos \frac{b_{i j}}{|\chi(\varphi)|}\right)+h\left(2 \pi-\arccos \frac{b_{i j}}{|\chi(\varphi)|}\right) .
$$

Hence, $h(\cdot)=0$ if the parallelogram is empty.

For the value of $\varphi$ such that $|\chi(\varphi)|=y$, we have $h\left(\varphi_{y}\right)=h\left(\varphi_{\chi}\right)$. Therefore, we obtain

$$
\begin{aligned}
F(y)=1-\frac{1}{\sum_{i=1}^{n}\left|a_{i}\right|} & \left(\sum_{i<j}^{I} \frac{y}{\sin \gamma_{i j}} \int_{\Phi_{i j}(y)} \sin \varphi \sin \left(\gamma_{i j}-\varphi\right) \mathrm{d} \varphi\right. \\
& \left.-\sum_{i<j}^{I I} I_{i j}(y) h\left(\varphi_{y}\right) \frac{\sqrt{y^{2}-b_{i j}^{2}}}{b_{i j}}+\sum_{i=1}^{n}\left(\left|a_{i}\right|-y\right)^{+}\right) .
\end{aligned}
$$

In the case where $\partial \mathbb{D}$ contains no pairs of parallel sides, (3.3) coincides with the expression given in [2, p. 158].

It follows from (3.3) that to find the distribution function $F(y)$ we have to calculate integrals of the form

$$
\frac{1}{\sin \gamma} \int_{\Phi(y)} \sin \varphi \sin (\gamma-\varphi) \mathrm{d} \varphi
$$

for any two nonparallel segments $a$ and $b(a \leq b)$, where $\gamma$ is the angle between $a$ and $b$ (or their continuations), and also calculate the second sum in (3.3) (for pairs of parallel sides). Here and below,

$\Phi(y)=\{\varphi$ : a chord joining $a$ and $b$ exists with direction $\varphi$ and length $y\}$. 


\section{The case of a regular polygon}

Consider a particular case of (3.3), where $\mathbb{D}$ is a regular polygon. We assume that edges of a regular polygon are ordered in the clockwise direction on $\partial \mathbb{D}$. We denote by $\gamma_{k}$ the angle between $a_{1}$ and $a_{k}$ (or their continuations), by $d_{k}$ the length of the segment connecting the end of side $a_{1}$ with the beginning of $a_{k}$, and by $h_{k}$ the distance between the beginning of segment $a_{k}$ and the line containing the segment $a_{1}$.

Below we will use the following notation:

$$
N=\left\{\begin{array}{ll}
n+\frac{1}{2} & \text { for a }(2 n+1) \text {-gon, } \\
n & \text { for a }(2 n) \text {-gon, }
\end{array} \text { and } \mathbf{1}(N)= \begin{cases}0 & \text { if } N=n+\frac{1}{2} \\
1 & \text { if } N=n\end{cases}\right.
$$

Consider a regular polygon. It is not difficult to verify that

$$
\begin{gathered}
\gamma_{k}=\frac{N-k+1}{N} \pi, \quad k=2, \ldots,\lfloor N\rfloor+1, \quad \gamma_{k}=\gamma_{2(N+1)-k}, \quad k=\lfloor N\rfloor+2, \ldots, 2 N, \\
d_{2}=0, \quad d_{3}=a, \quad d_{k}=d_{k-2}+2 a \sin \frac{\gamma_{k-2}}{2}, \quad k=4, \ldots,\lfloor N\rfloor+2, \\
d_{k}=d_{2(N+2)-k}, \quad k=\lfloor N\rfloor+3, \ldots, 2 N, \quad h_{k}=d_{k} \cos \frac{\gamma_{k}}{2},
\end{gathered}
$$

where $a$ is the length of the side of the polygon and $\lfloor N\rfloor$ is the floor function.

In this particular case, (3.3) can be rewritten as

$$
\begin{aligned}
F(y)= & 1-\frac{y}{a} \sum_{k=2}^{\lceil N\rceil} \frac{1}{\sin \gamma_{k}} \int_{\Phi_{1 k}(y)} \sin \varphi \sin \left(\gamma_{k}-\varphi\right) \mathrm{d} \varphi-\frac{1}{a}(a-y)^{+} \\
& +\frac{1}{a} \mathbf{1}(N) \mathbf{1}\left(d_{n+1} \leq y \leq d_{n+2}\right) \frac{\sqrt{y^{2}-d_{n+1}^{2}}\left(a-\sqrt{y^{2}-d_{n+1}^{2}}\right)}{y},
\end{aligned}
$$

where $\lceil N\rceil$ is the ceiling function.

It follows from (4.1) that in order to obtain an explicit form for the distribution function $F(y)$ of a regular polygon, we have to find the domains $\Phi_{1 k}(y)$ and then calculate the integrals on the right-hand side of (4.1).

By definition we have

$$
\Phi_{1 k}(y)=\left\{\varphi \text { : a chord joining } a_{1} \text { and } a_{k} \text { exists with direction } \varphi \text { and length } y\right\} .
$$

Below we use the notation

$$
\theta_{k}=\arctan \left(\frac{a}{h_{k}}+\tan \frac{\gamma_{k}}{2}\right)
$$

Note that if $k=2$, we have $h_{2}=0$ and, therefore, $\theta_{2}=\arctan (+\infty)=\pi / 2$.

The line $g$ with parameters $(\varphi, p)$ intersects the sides $a_{1}$ and $a_{k}$ if $\varphi \in\left(\gamma_{k}-\theta_{k}, \theta_{k}\right)$. Without loss of generality, we can assume that the reference direction ( $x$-axis) coincides with the direction of the segment $a_{1}$ and the origin $O$ coincides with the intersection of the lines containing $a_{1}$ and $a_{k}$. For the intersection point of the line $g=(\varphi, p)$ with the segment $a_{1}$, we have

$$
x=\frac{p}{\cos \varphi} \quad \text { and } \quad y=0
$$


Therefore,

$$
g \cap a_{1}=\left\{\begin{array}{l}
x=\frac{p}{\cos \varphi}, \\
y=0, \\
\frac{d_{k}}{2 \sin \left(\gamma_{k} / 2\right)} \cos \varphi \leq p \leq\left(a+\frac{d_{k}}{2 \sin \left(\gamma_{k} / 2\right)}\right) \cos \varphi .
\end{array}\right.
$$

For the intersection point of the line $g=(\varphi, p)$ with the segment $a_{k}$, we have

$$
x \cos \varphi+y \sin \varphi=p \quad \text { and } \quad y=\tan \gamma_{k} x
$$

or

$$
x=\frac{p \cos \gamma_{k}}{\cos \left(\varphi-\gamma_{k}\right)} \quad \text { and } \quad y=\frac{p \sin \gamma_{k}}{\cos \left(\varphi-\gamma_{k}\right)}
$$

Hence, we obtain

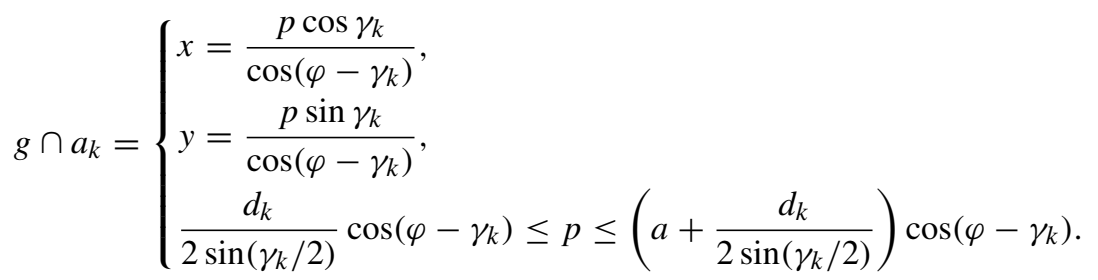

The line $g$ which intersects the sides $a_{1}$ and $a_{k}$ forms a chord $\chi$ of length

$$
|\chi|=\frac{p \sin \gamma_{k}}{\cos \varphi \cos \left(\varphi-\gamma_{k}\right)}
$$

Therefore, we obtain the following system of relations:

$$
\begin{aligned}
\frac{d_{k}}{2 \sin \left(\gamma_{k} / 2\right)} \cos \varphi & \leq p \leq\left(a+\frac{d_{k}}{2 \sin \left(\gamma_{k} / 2\right)}\right) \cos \varphi, \\
\frac{d_{k}}{2 \sin \left(\gamma_{k} / 2\right)} \cos \left(\varphi-\gamma_{k}\right) & \leq p \leq\left(a+\frac{d_{k}}{2 \sin \left(\gamma_{k} / 2\right)}\right) \cos \left(\varphi-\gamma_{k}\right), \\
|\chi| & =\frac{p \sin \gamma_{k}}{\cos \varphi \cos \left(\varphi-\gamma_{k}\right)} .
\end{aligned}
$$

If $\cos \varphi>\cos \left(\varphi-\gamma_{k}\right)$ then $\sin \varphi \sin \gamma_{k}<\left(1-\cos \gamma_{k}\right) \cos \varphi$. Hence, $\tan \varphi \leq \tan \gamma_{k} / 2$. Therefore, $\varphi \in\left(\gamma_{k}-\theta_{k}, \gamma_{k} / 2\right)$.

Since $\varphi \in\left(\gamma_{k}-\theta_{k}, \gamma_{k} / 2\right)$, then (4.2a)-(4.2c) have the following form:

$$
\begin{gathered}
\frac{d_{k}}{2 \sin \left(\gamma_{k} / 2\right)} \cos \varphi \leq p \leq\left(a+\frac{d_{k}}{2 \sin \left(\gamma_{k} / 2\right)}\right) \cos \left(\varphi-\gamma_{k}\right), \\
|\chi|=\frac{p \sin \gamma_{k}}{\cos \varphi \cos \left(\varphi-\gamma_{k}\right)} .
\end{gathered}
$$

Moreover, we obtain

$$
\chi_{\min }(\varphi)=\frac{h_{k}}{\cos \left(\varphi-\gamma_{k}\right)}, \quad \chi_{\max }(\varphi)=\frac{h_{k+1}}{\cos \varphi} .
$$


The function $\chi_{\min }(\varphi)$ decreases in the domain $\varphi \in\left(\gamma_{k}-\theta_{k}, \gamma_{k} / 2\right)$, and

$$
\varphi_{\max }=\gamma_{k}-\theta_{k}, \quad \varphi_{\min }=\frac{\gamma_{k}}{2}, \quad \chi_{\min }\left(\varphi_{\max }\right)=d_{k+1}, \quad \text { and } \quad \chi_{\min }\left(\varphi_{\min }\right)=d_{k} .
$$

If $\gamma_{k}<\theta_{k}$ then the function $\chi_{\max }(\varphi)$ decreases in the domain $\varphi \in\left(\gamma_{k}-\theta_{k}, 0\right)$ and increases in the domain $\varphi \in\left(0, \gamma_{k} / 2\right)$. Hence, we have

$\varphi_{\min }=0, \quad \chi_{\max }\left(\gamma_{k}-\theta_{k}\right)=d_{k+1}, \quad \chi_{\max }\left(\varphi_{\min }\right)=h_{k+1}, \quad$ and $\quad \chi_{\max }\left(\frac{\gamma_{k}}{2}\right)=d_{k+2}$.

If $\gamma_{k} \geq \theta_{k}$ then the function $\chi_{\max }(\varphi)$ increases in the domain $\varphi \in\left(\gamma_{k}-\theta_{k}, \gamma_{k} / 2\right)$. In this case,

$$
\varphi_{\min }=\gamma_{k}-\theta_{k}, \quad \varphi_{\max }=\frac{\gamma_{k}}{2}, \quad \chi_{\max }\left(\varphi_{\min }\right)=d_{k+1}, \quad \text { and } \quad \chi_{\max }\left(\varphi_{\max }\right)=d_{k+2} .
$$

Now let $\varphi \in\left(\gamma_{k} / 2, \theta_{k}\right)$. In this case, (4.2a)-(4.2c) can be rewritten in the form

$$
\begin{gathered}
\frac{d_{k}}{2 \sin \left(\gamma_{k} / 2\right)} \cos \left(\varphi-\gamma_{k}\right) \leq p \leq\left(a+\frac{d_{k}}{2 \sin \left(\gamma_{k} / 2\right)}\right) \cos \varphi \\
|\chi|=\frac{p \sin \gamma_{k}}{\cos \varphi \cos \left(\varphi-\gamma_{k}\right)} .
\end{gathered}
$$

Moreover, we obtain

$$
\chi_{\min }(\varphi)=\frac{h_{k}}{\cos \varphi}, \quad \chi_{\max }(\varphi)=\frac{h_{k+1}}{\cos \left(\varphi-\gamma_{k}\right)} .
$$

The function $\chi_{\min }(\varphi)$ increases in the domain $\left(\gamma_{k} / 2, \theta_{k}\right)$ and

$$
\varphi_{\max }=\theta_{k}, \quad \varphi_{\min }=\frac{\gamma_{k}}{2}, \quad \chi_{\min }\left(\varphi_{\max }\right)=d_{k+1}, \quad \text { and } \quad \chi_{\min }\left(\varphi_{\min }\right)=d_{k} .
$$

If $\gamma_{k}<\theta_{k}$ then the function $\chi_{\max }(\varphi)$ decreases in the domain $\left(\gamma_{k} / 2, \gamma_{k}\right)$ and increases in the domain $\left(\gamma_{k}, \theta_{k}\right)$. Hence, we obtain

$$
\varphi_{\min }=\gamma_{k}, \quad \chi_{\max }\left(\varphi_{\min }\right)=h_{k+1}, \quad \chi_{\max }\left(\theta_{k}\right)=d_{k+1}, \quad \text { and } \quad \chi_{\max }\left(\frac{\gamma_{k}}{2}\right)=d_{k+2} .
$$

If $\gamma_{k} \geq \theta_{k}$ then the function $\chi_{\max }(\varphi)$ decreases in the domain $\left(\gamma_{k} / 2, \theta_{k}\right)$ and we have

$$
\varphi_{\max }=\frac{\gamma_{k}}{2}, \quad \varphi_{\min }=\theta_{k}, \quad \chi_{\max }\left(\varphi_{\min }\right)=d_{k+1}, \quad \text { and } \quad \chi_{\max }\left(\varphi_{\max }\right)=d_{k+2} .
$$

Therefore, in the case in which $\gamma_{k}<\theta_{k}$ we have

$$
\Phi_{1 k}(y)= \begin{cases}\left(\gamma_{k}-\arccos \frac{h_{k}}{y}, \arccos \frac{h_{k}}{y}\right) & \text { if } y \in\left[d_{k}, h_{k+1}\right), \\ \left(\gamma_{k}-\arccos \frac{h_{k}}{y},-\arccos \frac{h_{k+1}}{y}\right) & \\ \cup\left(\arccos \frac{h_{k+1}}{y}, \gamma_{k}-\arccos \frac{h_{k+1}}{y}\right) & \\ \cup\left(\gamma_{k}+\arccos \frac{h_{k+1}}{y}, \arccos \frac{h_{k}}{y}\right) & \text { if } y \in\left[h_{k+1}, d_{k+1}\right), \\ \left(\arccos \frac{h_{k+1}}{y}, \gamma_{k}-\arccos \frac{h_{k+1}}{y}\right) & \text { if } y \in\left[d_{k+1}, d_{k+2}\right], \\ \varnothing & \text { if } y>d_{k+2} \text { or } y<d_{k},\end{cases}
$$


and if $\gamma_{k} \geq \theta_{k}$, we have

$$
\Phi_{1 k}(y)= \begin{cases}\left(\gamma_{k}-\arccos \frac{h_{k}}{y}, \arccos \frac{h_{k}}{y}\right) & \text { if } y \in\left[d_{k}, d_{k+1}\right), \\ \left(\arccos \frac{h_{k+1}}{y}, \gamma_{k}-\arccos \frac{h_{k+1}}{y}\right) & \text { if } y \in\left[d_{k+1}, d_{k+2}\right], \\ \varnothing & \text { if } y>d_{k+2} \text { or } y<d_{k} .\end{cases}
$$

Now we can substitute the values of $\Phi_{1 k}(y)$ into the right-hand side of (4.1) and calculate the corresponding integrals.

Therefore, the distribution function for a regular polygon has the following form:

$$
\begin{aligned}
& F(y)=1-\frac{y}{a} \sum_{k=2}^{\lceil N\rceil}\left\{\mathbf { 1 } ( \gamma _ { k } < \theta _ { k } ) \left[\mathbf { 1 } ( y \in [ d _ { k } , h _ { k + 1 } ) ) \left(\left[\frac{h_{k}}{y} \sqrt{1-\frac{h_{k}^{2}}{y^{2}}}-\arccos \frac{h_{k}}{y}+\frac{\gamma_{k}}{2}\right] \cot \gamma_{k}\right.\right.\right. \\
& \left.+\frac{1}{2}-\frac{h_{k}^{2}}{y^{2}}\right) \\
& +\mathbf{1}\left(y \in\left[h_{k+1}, d_{k+1}\right)\right) \\
& \times\left(\left[\frac{h_{k}}{y} \sqrt{1-\frac{h_{k}^{2}}{y^{2}}}-2 \frac{h_{k+1}}{y} \sqrt{1-\frac{h_{k+1}^{2}}{y^{2}}}-\arccos \frac{h_{k}}{y}\right.\right. \\
& \left.\left.+2 \arccos \frac{h_{k+1}}{y}+\frac{\gamma_{k}}{2}\right] \cot \gamma_{k}+\frac{1}{2}-\frac{h_{k}^{2}}{y^{2}}\right) \\
& +\mathbf{1}\left(y \in\left[d_{k+1}, d_{k+2}\right)\right) \\
& \times\left(\left[\arccos \frac{h_{k+1}}{y}-\frac{h_{k+1}}{y} \sqrt{1-\frac{h_{k+1}^{2}}{y^{2}}}-\frac{\gamma_{k}}{2}\right] \cot \gamma_{k}\right. \\
& \left.\left.-\frac{1}{2}+\frac{h_{k+1}^{2}}{y^{2}}\right)\right] \\
& +\mathbf{1}\left(\gamma_{k} \geq \theta_{k}\right)\left[\mathbf{1}\left(y \in\left[d_{k}, d_{k+1}\right)\right)\right. \\
& \times\left(\left[\frac{h_{k}}{y} \sqrt{1-\frac{h_{k}^{2}}{y^{2}}}-\arccos \frac{h_{k}}{y}+\frac{\gamma_{k}}{2}\right] \cot \gamma_{k}+\frac{1}{2}-\frac{h_{k}^{2}}{y^{2}}\right) \\
& +\mathbf{1}\left(y \in\left[d_{k+1}, d_{k+2}\right)\right) \\
& \times\left(\left[\arccos \frac{h_{k+1}}{y}-\frac{h_{k+1}}{y} \sqrt{1-\frac{h_{k+1}^{2}}{y^{2}}}-\frac{\gamma_{k}}{2}\right] \cot \gamma_{k}\right. \\
& \left.\left.\left.-\frac{1}{2}+\frac{h_{k+1}^{2}}{y^{2}}\right)\right]\right\}-\frac{1}{a}(a-y)^{+} \\
& +\frac{1}{a} \mathbf{1}(N) \mathbf{1}\left(d_{n+1} \leq y \leq d_{n+2}\right) \frac{\sqrt{y^{2}-d_{n+1}^{2}}\left(a-\sqrt{y^{2}-d_{n+1}^{2}}\right)}{y} \text {. }
\end{aligned}
$$


It is not difficult to verify that this function is a continuous function, and, for a regular triangle, a square, a regular pentagon, and a regular hexagon, (4.2) coincides with those of [11], [6], [3], and [9], respectively.

From the above formulae, it is not difficult to specify a chord length density function $f(y)=$ $F^{\prime}(y)$ for regular polygons.

\section{Acknowledgement}

We are grateful for the careful reading and some helpful comments of an anonymous referee.

\section{References}

[1] Ambartzumian, R. V. (1982). Combinatorial Integral Geometry. John Wiley, Chichester.

[2] Ambartzumian, R. V. (1990). Factorization Calculus and Geometric Probability (Encyclopaedia Math. Appl. 33). Cambridge University Press.

[3] Aharonyan, N. G. and Ohanyan, V. K. (2005). Chord length distribution functions for polygons. J. Contemp. Math. Anal. 40, 43-56.

[4] Gates, J. (1982). Recognition of triangles and quadrilaterals by chord length distribution. J. Appl. Prob. 19, 873-879.

[5] Gates, J. (1987). Some properties of chord length distributions. J. Appl. Prob. 24, 863-874.

[6] Gille, W. (1988). The chord length distribution of parallelepipeds with their limiting cases. Exp. Techn. Phys. 36, 197-208.

[7] Gille, W. (2003). Cross-section structure functions in terms of the three-dimensional structure functions of infinitely long cylinders. Powder Tech. 138, 124-131.

[8] Gille, W., Aharonyan, N. G. and Haruttyunyan, H. S. (2009). Chord length distribution of pentagonal and hexagonal rods: relation to small-angle scattering J. Appl. Crystallography 42, 326-328.

[9] Harutyunyan, H. (2007). Chord length distribution function for regular hexagon. Uchenie Zapiski, Yerevan State University 1, 17-24.

[10] ShIlov, G. (1984). Mathematical Analysis, 2nd edn. Moscow State University.

[11] Sulanke, R. (1961). Die Verteilung der Sehnenlängen an ebenen und räumlichen Figuren. Math. Nachr. 23, 51-74.

[12] Stoyan, D. and Stoyan, H. (1994). Fractals, Random Shapes and Point Fields. John Wiley, Chichester.

[13] Sukiasian, H. S. and Gille, W. (2007). Relation between the chord length distribution of an infinitely long cylinder and that of its base. J. Math. Phys. 48, 8 pp. 